\section{Prävention: Vor Depression schützen}

Psychologische Präventionsprogramme können die Zahl depressiver Neuerkrankungen eindämmen. Zu diesem Ergebnis sind niederländische Forscher gekommen, nachdem sie in einer Metaanalyse die Daten von insgesamt 32 Studien mit 6214 Teilnehmern ausgewertet hatten. Personen ohne Depression, die an Präventionsprogrammen teilgenommen hatten, entwickelten bis zum Studienende seltener depressive Episoden als die Teilnehmer der Kontrollgruppe (Inzidenz Rate Ratio: 0,79 , Number needed to treat: 20). In den Subgruppenanalysen deutete sich an, dass die interpersonelle Psychotherapie effektiver war als eine kognitive Verhaltenstherapie.

Int J Epidemiol 2014; 43; 318-329

\section{DATEN UND FAKTEN}

\subsection{6}

Menschen nahmen sich im Jahr 2013 in Deutschland das Leben, die meisten von ihnen aufgrund von Depressionen. Damit ist die Zahl der Suizide fast dreimal so hoch wie die Zahl der Verkehrstoten. Besorgniserregend ist außerdem, dass die Anzahl der Suizidversuche etwa sieben- bis zwölfmal höher ist als die der vollzogenen Suizide. Am stärksten gefährdet sind offenbar Männer: In ihrer Gruppe nehmen sich mehr als doppelt so viele das Leben wie in der Gruppe der Frauen.

\section{INFOS IM INTERNET}

Die S3-Leitline (Nationale Versorgungsleitlinie) „Unipolare Depression“ mit Empfehlungen zur Diagnostik und Therapie ist im Internet einsehbar auf: www.leitlinien.de/nvl/depression

Die Stiftung Deutsche Depressionshilfe bietet auf ihren Webseiten umfangreiche Informationen zu Depression, angefangen von der Forschung bis hin zur Versorgung der Patienten: www.deutsche-depressionshilfe.de

Rat und Fortbildungen zum Thema Depression und zu anderen psychiatrischen Erkrankungen finden Ärzte, Psychologen und Angehörige anderer Berufe im Gesundheitswesen unter der Internet-Adresse: www.psychiatriekonsil.de

Komorbiditäten erhöhen Suizidrate

\title{
Angst und Panik sind häufige Begleiter
}

Depressive Episoden treten oft gleichzeitig mit anderen psychischen oder somatischen Erkrankungen auf. Daran sollte man bei der diagnostischen Abklärung denken.

Fast jeder zweite depressive Patient hat zusätzlich Angst- und Panikstörungen. Auch Alkohol-, Medikamenten- und Drogenabhängigkeit sind bei solchen Patienten keine Seltenheit. Umgekehrt leiden 30-60\% der Patienten mit Suchterkrankungen gleichzeitig an depressiven Störungen. Außerdem treten Essstörungen, somatoforme Störungen, Persönlichkeitsstörungen und Zwangsstörungen oftmals gemeinsam mit depressiven Störungen auf.

Diese Komorbiditäten sind insofern von Bedeutung, da sie bei den meisten $\mathrm{Pa}$ tienten mit funktionellen Beschwerden, einer stärkeren depressiven Symptomatik und einer höheren Suizidrate einhergehen. Psychische Begleiterkrankungen sollten daher auf jeden Fall abgeklärt werden, weil sie sowohl die Behandlung als auch die Prognose der depressiven Grunderkrankung erschweren.

Die Patienten sprechen insgesamt schlechter auf die medikamentöse oder psychotherapeutische Behandlung an. Eine psychologische Zusatzdiagnostik ist aber nicht per se indiziert. Ob eine auf andere Störungen gerichtete Diagnostik indiziert ist, hängt vom Vorliegen konkreter Verdachtsmomente ab.

\section{Risiko für somatische Erkrankungen}

Außerdem haben depressive Patienten ein erhöhtes Risiko für somatische Erkrankungen. Einer WHO-Studie zufolge ist das Risiko für eine körperliche Beeinträchtigung im Jahr nach einer depressiven Erkrankung um das 1,8-Fache erhöht.

Dieser Zusammenhang ist zum einen darin begründet, dass schwere körperliche Erkrankungen mit psychischen Belastungen verbunden sein können. Zum anderen kann auch die depressive Erkrankung selbst erhebliche Auswirkungen auf den körperlichen Allgemeinzustand des Betroffenen haben. Die Folgen können Schlafstörungen, Erschöpfung oder allgemeine Schwäche sein, bis hin zu anderen chronischen somatischen Erkrankungen wie Diabetes, Arthritis und Bluthochdruck. Deshalb sollte auch das Vorliegen von körperlichen Erkrankungen sowie die Einnahme von Medikamenten und Noxen, die mit depressiven Symptomen einhergehen können, sorgfältig geprüft werden. (jn)

www.aerztezeitung.de

\section{Studien belegen Effekt}

\section{Sport gegen Depression}

Regelmäßige sportliche Aktivitäten sind auch für Depressive empfehlenswert, wie Studien zeigen.

Metaanalysen bestätigen den antidepressiven Effekt von Ausdauertraining und anderen Formen körperlicher Aktivität wie Krafttraining. Einer Cochrane-Analyse zufolge scheint die antidepressive Wirkung sportlicher Aktivitäten ähnlich groß zu sein wie der Effekt einer Psycho- oder
Pharmakotherapie. Die Interventionen beinhalteten Gehen, Laufen, Fahrradfahren, Tanzen, Rudern und Krafttraining. Auch Langzeiteffekte, die über die Dauer der Intervention hinausgingen, wurden nachgewiesen, aber mit geringer Effektstärke. Am effektivsten scheinen Krafttraining und Interventionen mit höherer Intensität und längerer Dauer zu sein.

(jn) 